\title{
Evolutionary rate patterns of genes involved in the Drosophila Toll and Imd signaling pathway
}

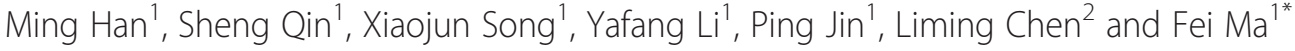

\begin{abstract}
Background: To survive in a hostile environment, insects have evolved an innate immune system to defend against infection. Studies have shown that natural selection may drive the evolution of immune system-related proteins. Yet, how network architecture influences protein sequence evolution remains unclear. Here, we analyzed the molecular evolutionary patterns of genes in the Toll and Imd innate immune signaling pathways across six Drosophila genomes within the context of a functional network.

Results: Based on published literature, we identified 50 genes that are directly involved in the Drosophila Toll and Imd signaling pathways. Of those genes, only two (Sphinx1 and Dnr1) exhibited signals of positive selection. There existed a negative correlation between the strength of purifying selection and gene position within the pathway; the downstream genes were more conserved, indicating that they were subjected to stronger evolutionary constraints. Interestingly, there was also a significantly negative correlation between the rate of protein evolution and the number of regulatory microRNAs, implying that genes regulated by more miRNAs experience stronger functional constraints and therefore evolve more slowly.
\end{abstract}

Conclusion: Taken together, our results suggested that both network architecture and miRNA regulation affect protein sequence evolution. These findings improve our understanding of the evolutionary patterns of genes involved in Drosophila innate immune pathways.

Keywords: Drosophila, Toll and Imd signaling pathway, Molecular evolution, Selective constraint, microRNA

\section{Background}

Over the past few years, molecular evolution within a network architecture has been of great interest in population genetics [1-7]. Studies on major cellular pathways have demonstrated that network topology constrains the evolutionary pattern of genes. Many reports have demonstrated the existence of a positive correlation between gene pathway position and nucleotide substitution rate. For example, in the melanin synthesis pathway of silkworms [8], the anthocyanin biosynthetic pathway in plants [9-11], and the Drosophila Ras signal transduction pathway [12] the upstream genes are subjected to stronger evolutionary constraints. In contrast, an opposite effect was observed in other pathways, such as the animal Tolllike receptor (TLR) [5] and the yeast HOG [13] signaling

\footnotetext{
* Correspondence: mafei01@tsinghua.org.cn

'Laboratory for Comparative Genomics and Bioinformatics \& Jiangsu Key Laboratory for Biodiversity and Biotechnology, College of Life Science, Nanjing Normal University, Nanjing 210023, P. R China

Full list of author information is available at the end of the article
}

pathways. Moreover, in the Caenorhabditis insulin/TOR signaling transduction pathway, the pattern of selective constraints is driven by expression level [1], whereas in the $\mathrm{N}$-glycosylation metabolic pathways across primates, connectivity of each gene drives the strength of purifying selection [2].

Multiple factors may affect the evolution of genes within networks and pathways, including the gene position, gene expression level [14-16], protein length [17], codon bias $[7,18]$, connectivity $[19,20]$, the number of regulatory microRNAs (miRNAs) that target a gene [21], and the length of its 3 '-untranslated region ( 3 '-UTR) $[22,23]$. Furthermore, studies have demonstrated that miRNAs participate in the regulation of innate immunity and inflammatory responses $[24,25]$. Thus, miRNA regulation should be analyzed from an evolutionary perspective to improve our understanding of the impact of miRNAs on protein evolution.

The nuclear factor $\kappa \mathrm{B}(\mathrm{NF}-\kappa \mathrm{B})$ pathway plays a central role in innate immunity by which invertebrates defend

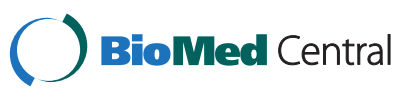

(c) 2013 Han et al.; licensee BioMed Central Ltd. This is an open access article distributed under the terms of the Creative Commons Attribution License (http://creativecommons.org/licenses/by/2.0), which permits unrestricted use, distribution, and reproduction in any medium, provided the original work is properly cited. 
against pathogens [26,27]. Drosophila possess two pathways (Toll and Imd innate immune response system; Figure 1) to activate NF- $\mathrm{B}$ transcription factors $[27,28]$. The Toll pathway is responsible for defense against Gram-positive bacteria or fungi when the cleaved ligand Spatzle binds to the Toll receptor, eventually leading to the activation of the NF- $\mathrm{kB}$ family members Dorsal and Dorsal-related immunity factor (Dif). This pathway also participates both in developmental processes [29-31] and immunity $[32,33]$. In contrast, the Imd pathway controls resistance to Gram-negative bacterial infections $[32,34]$. The JAK-STAT pathway also correlates with the Drosophila innate immune response, but unlike the Toll and Imd pathways, it remains poorly understood, and only four genes (UPD, DOME, JAK, STAT) have been reported within this pathway [34-36]. Studies have shown that natural selection may drive the evolution of immune system proteins [37-39]. Yet, the mechanism by which network architecture influences protein evolution within innate immune systems remains unclear. The complete genome sequences of Drosophila species and our current knowledge of the innate immune pathways in D. melanogaster together offer opportunity for a fine-scale evolutionary analysis of the Drosophila Toll and Imd pathways within the context of a network framework.

By surveying recent papers, we identified 50 immunerelated genes that are directly involved in the Drosophila
Toll and Imd signaling pathways, by transferring signals from receptor to transcription factor. We further investigated the evolutionary mechanism of Toll and Imd pathway genes across Drosophila species to understand 1) whether there exists a correlation between the strength of purifying selection and gene pathway position within the Toll and Imd innate immune signaling pathways; and 2) which of the topological parameters that characterize network evolution contributes most to the observed selective patterns.

\section{Results}

\section{Analysis of protein sequence evolution}

Variation in selective constraints across immunity pathways was assessed with the help of the program PAML [40] and a comparison of alternative evolutionary models. For all 50 immune-related genes that we identified from the literature, the M0 (one $d N / d S$ ratio) model calculated a single nonsynonymous/synonymous substitution rate $(\omega=d N / d S)$ ratio for all branches using a MUSCLE alignment [41]. According to our results, the Drosophila immune genes have undergone strong functional constraints, with $\omega$ values ranging from 0.0001 (eff) to 0.4290 (Spz) (Additional file 1: Table S1). Additionally, by comparing the M1a (nearly neutral) and M2a (positive selection) models, the M7 (beta) and M8 (beta \& $\omega$ ) models, and also the M8 and M8a (beta \& $\omega_{s}=1$ ) models,

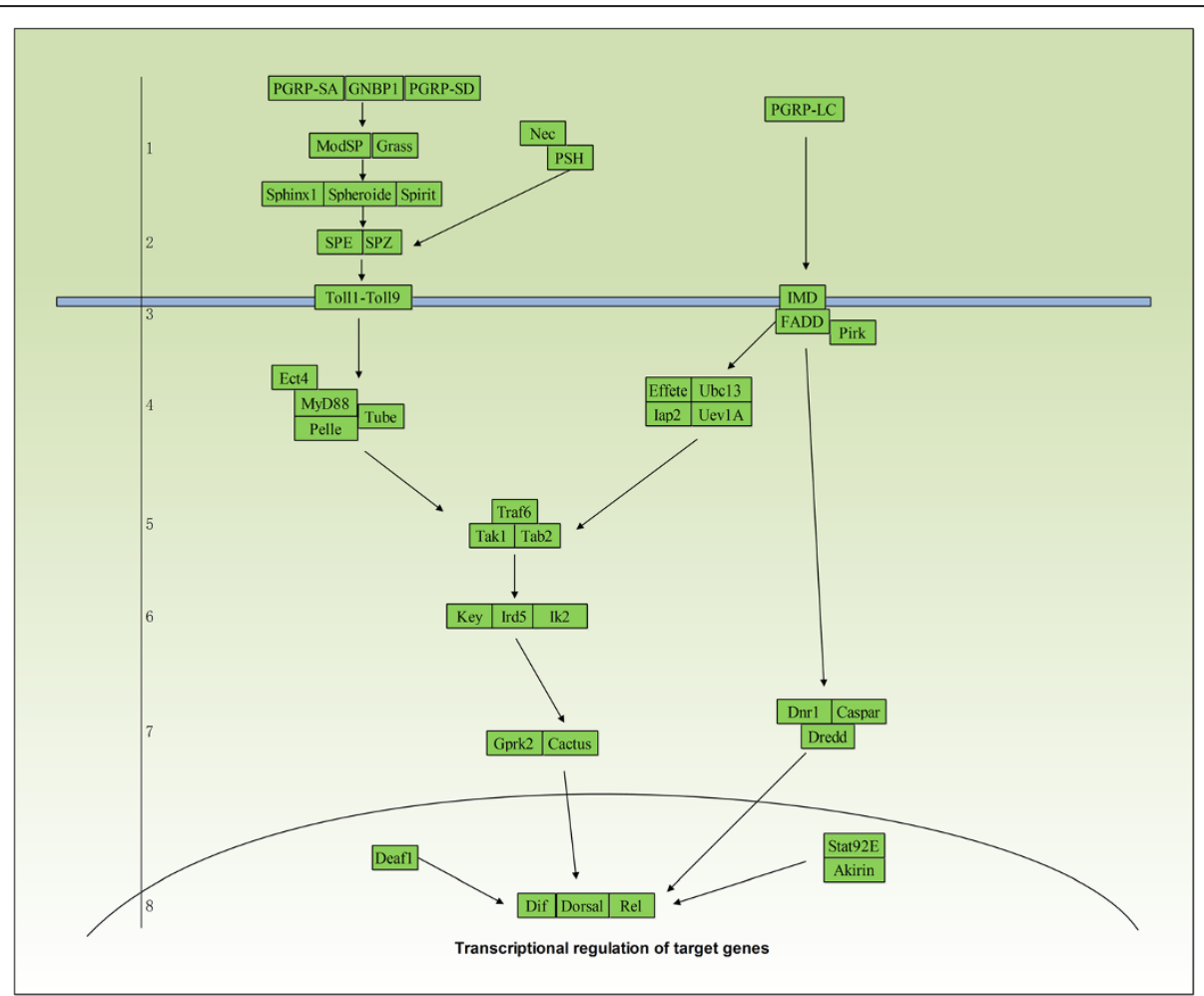

Figure 1 The Drosophila Toll and Imd pathways. 
positive selection was observed for Spz, Mstprox, $T l$, Toll-4, cact, Dif, Sphinx1, Ect4, and Dnr1 (Additional file 1: Table S2). However, after the false discovery rate test $(q=0.05)$, only five genes (Dnr1, Sphinx1, Dif, Cact and Ect4) remained significantly positively selected (Additional file 1: Table S2). To improve the reliability of our analysis, we further used a PRANK $[42,43]$ alignment and detected positive selection for Dnr1, Sphinx1, $M s t$ and $T l$ after the FDR test $(q=0.05)$ (Additional file 1: Table S3). The PAML results based on two different alignments (MUSCLE and PRANK) were only consistent for two genes, Sphinx1 and Dnr1. Therefore, these two genes seemed to contain a rather robust signal of positive selection, strongly suggesting that they were indeed subjected to positive selective constraints.

\section{The strength of purifying selection within Drosophila Toll and Imd signaling pathways}

Correlations between evolutionary parameters $(d S, d N$, and $\omega$ ) and topological factors (i.e., gene position within the pathway, gene expression level, protein length, codon usage bias, connectivity of individual genes, the number of regulatory miRNAs, the length of 3 '-UTR) were estimated applying Spearman's rank correlation coefficients $(\rho)$. For different factors that may affect protein sequence evolution, a significant negative correlation between $\omega$ values and gene position was observed $(\rho=-0.370[-0.529,-0.114], P=0.020$ after FDR correction; Figure 2 and Additional file 1: Table S4), indicating that the downstream genes were more conserved and therefore underwent stronger purifying selection. In addition, $d N$ and $d S$ values were significantly correlated with gene position $(d N: \rho=-0.476$ $[-0.649,-0.243], P=0.004$ after FDR correction; $d S$ : $\rho=-0.467[-0.645,-0.237], P=0.004$ after FDR correction; Figure 2 and Additional file 1: Table S4).

Given that selective constraints can be affected by various topological factors, the relationship between different variables was further analyzed. According to our results, connectivity was significantly correlated with the expression level of immune genes after infection by bacterial (Exp1) or fungal (Exp2) (Exp1: $\rho=0.450$ [0.197, 0.671], $P=0.005$ after FDR correction; Exp2: $\rho=0.565$ [0.329, 0.737], $P<0.001$ after FDR correction; Additional file 1: Table S4). When we estimated the expression levels after infection at each separate time point, similar results were observed (Additional file 1: Table S5). The highly significant correlation between connectivity and gene expression level indicated that the more connected a protein was, the higher gene expression level it had.

We also observed that the number of regulatory miRNAs $\left(N_{m i R}\right.$, predicted using Miranda 3.3 [44] with default parameters) and the length of the $3^{\prime}$-UTR $\left(L_{3}{ }^{\prime}\right.$ UTR $)$ both significantly correlated with $d N, d S$, and $\omega$ (Figure 3 and Table 1). To test the robustness of our predicted miRNA targets, we set the Miranda score to a higher level of 150.0, 160.0, and the correlation remained significant (Additional file 1: Table S4). These results confirmed that genes regulated by more miRNAs were likely to undergo stronger functional constraints and therefore evolve at slower rates $[21,45]$.

\section{Multivariate analysis}

To investigate whether the observed correlations resulted from direct or indirect influences, two multivariate analysis techniques (partial analysis and path analysis) were performed. As shown in Additional file 1: Table S4, gene pathway position, the number of regulatory miRNAs, and the length of the $3^{\prime}$-UTR are correlated with each other and with the $d N, d S$, and $\omega$ values. Partial analysis revealed that, when controlling for $N_{m i R}$ and $L_{3}$ 'UTR, the correlation between position and $d N$ remained significant $(\rho=-0.318[-0.506,-0.168], P=0.028)$ while those between position and $d S$ and between position and $\omega$ disappeared $(d S: \rho=-0.203[-0.465,-0.076], P=0.167 ; \omega$ : $\rho=-0.233[-0.422,0.010], P=0.112)$. Similarly, when controlling for $L_{3}{ }^{\prime} U T R$, the significant correlations disappeared, except for those between $d N$ and position ( $\rho=-0.333[-0.504,-0.206], P=0.019)$, between $N_{m i R}$ and $d N(\rho=-0.384[-0.664,-0.044], P=0.007)$, and between $N_{m i R}$ and $d S(\rho=-0.361[-0.701,0.156], P=0.011)$, suggesting that the correlations between $N_{m i R}$ and $d N$ and between $N_{\text {miR }}$ and $d S$ were not mediated by the length of the 3 '-UTR. However, when controlling for $N_{m i R}$, only the correlation between $d N$ and position remained significant ( $\rho=-0.304[-0.486,-0.126], P=0.034)$, while the correlations between $L_{3}{ }^{\prime} U T R$ and $d N, d S$, and $\omega$ were no longer significant $(d N: \rho=0.072[-0.372,0.554], P=0.624 ; d S$ : $\rho=0.270[-0.373,0.670], P=0.060 ; \omega: \rho=-0.248[-0.439$, 0.011 ], $P=0.085$ ), indicating that the correlation between gene evolutionary rate and $L_{3}$ 'UTR was mediated by the number of regulatory miRNAs. Throughout our partial analysis, the significant correlation between pathway position and $d N$ consequently indicated that gene position within the pathway is an important parameter that influences protein sequence evolution within a network framework.

Through path analysis, the regression coefficients can be decomposed into direct and indirect correlations under the user-defined causal model. We therefore applied path analysis to investigate which contributor primarily constrained protein evolution within the network framework. During our path analysis (Figure 4), $d N$ and $\omega$ were considered endogenous variables while the other variables were considered exogenous. Consistent with the results of partial correlation analysis, we observed that $d N$ was significantly affected by position $(\beta=-0.306, P=0.020)$ while $\omega$ was only affected by $d N(\beta=0.418, P=0.008)$ even 


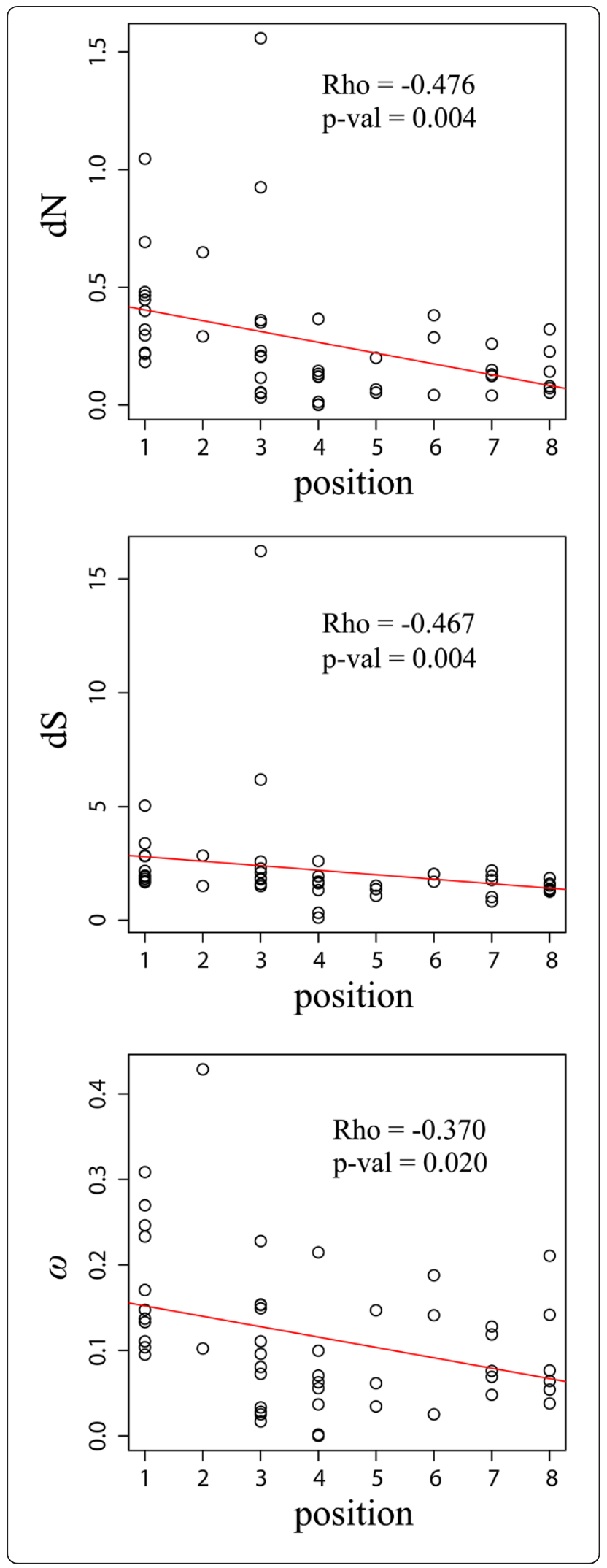

Figure 2 Nonsynonymous $(d N)$ and synonymous $(d S)$ substitution rates and their ratio $(\omega=d N / d S)$ as functions of pathway position. The significant negative correlation between $\omega$ and pathway position indicated that downstream genes were subjected to stronger purifying selection.

after removing the influence of other topological features. In addition, $d N$ was significantly correlated with ENC ( $\beta=0.405, P=0.002)$. Thus, Figure 4 demonstrates that pathway position and ENC can affect $\omega$ by influencing $d N$. Similar results were observed when ENC was considered as an endogenous variable in addition to $d N$ and $\omega$. Together, these results suggest that among the parameters that describe the network, pathway position is a major contributor to the tendency of the selective constraints.

\section{Discussion}

Positive selection acting on Toll and Imd pathway genes There were 40 genes in common between the study by Sackton et al. on the dynamic evolution of the Drosophila innate immune system [37] and ours. Sackton and his colleagues detected signals of positive selection for Dnr1, ModSP, PGRP-LC, and Nec, whereas we detected signals of positive selection for Dnr1, Sphinx1, Dif, Cact, and Ect4. The discrepancy between these studies might result from the use of different aligners (Sackton et al.: T-Coffee, us: MUSCLE). To explore the hypothesis, we performed the similarity analysis again using PRANK. Our results showed positive selection for Dnr1, Sphinx1, $M s t$, and $T l$ after the FDR test $(q=0.05)$. Only two of the genes, Sphinx1 and Dnrl, were consistently identified in at least two of the three analyses based on different alignments. Therefore, these two genes seemed to exhibit a rather robust signal of positive selection, strongly indicating that they have indeed undergone such selective constraints. Overall, the discrepancy between different alignments confirmed that the choice of alignment algorithm has a strong impact on estimates of positive selection [46]. As described in Markova-Raina et al., not all alignment errors are created equal, and even PRANK, which was reported to outperform other aligners in simulation [46], still has a high false positives rate; therefore, one must compare the results of different alignment programs to reduce the rate of false positives in estimates of positive selection.

For Sphinx1, which is located upstream in the innate immune pathway, the positive selection acting on it might make it more functional, better adapted to the changing extracellular environment, and subsequently, gain new functions through adaptive evolution $[38,47,48]$. Furthermore, a quantitative analysis by Obbard et al. [3] confirmed that adaptive evolution is a major factor driving molecular evolution within the Drosophila immune 

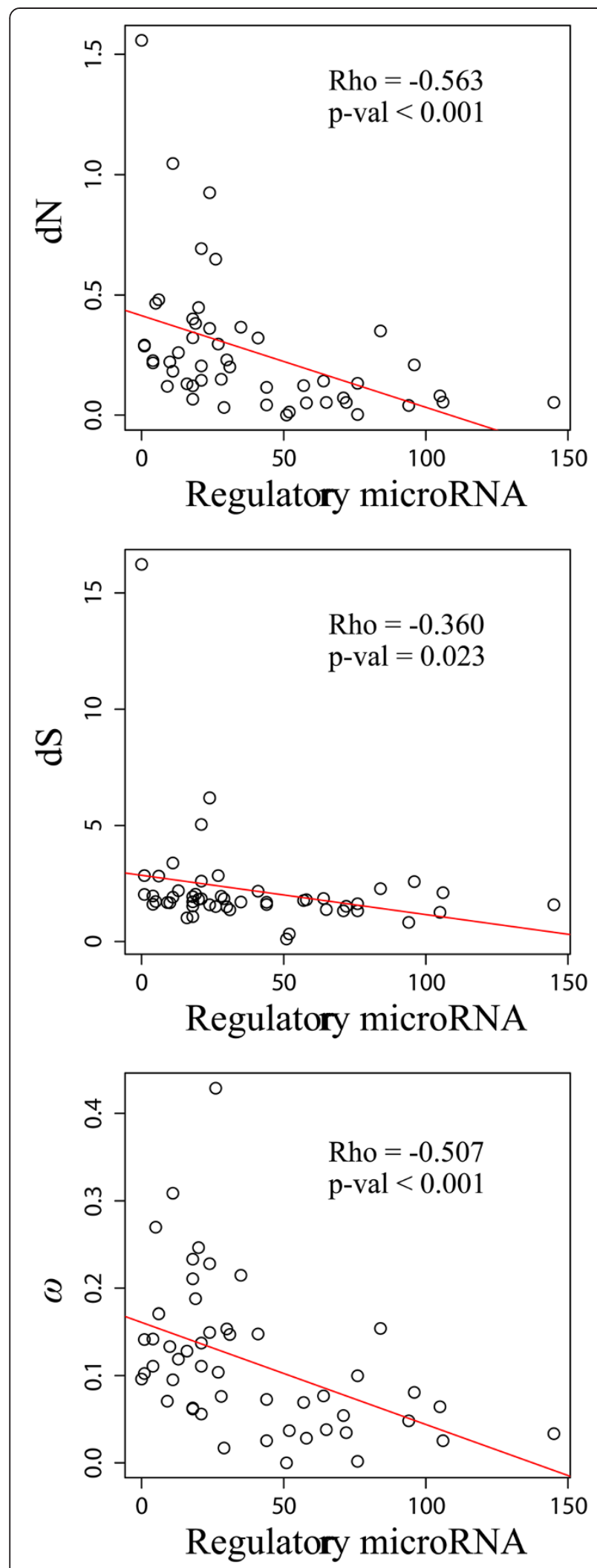

Figure 3 Nonsynonymous $(d N)$ and synonymous $(d S)$ substitution rates and their ratio $(\omega=d N / d S)$ as functions of the number of regulatory microRNAs. The significant negative correlation between $\omega$ and the number of regulatory miRNAs indicated that genes regulated by more miRNAs were more conserved and therefore evolved more slowly.

system. While we performed our network-level analysis from a phylogenetic perspective, Obbard et al. focused on population genetic data (for D. melanogaster and D. simulans) to quantify the effects of natural selection. Despite different data and methods in our study and that of Obbard et al., we both found that the peptidoglycan recognition protein and Gram-negative binding protein showed no signs of positive selection, perhaps because of their roles in binding with highly conserved microbial molecules [49].

\section{Selective constraints and pathway architecture}

Because upstream genes are more exposed to the hostile environment, to defend against pathogens, mutations in these genes are likely to have more pleiotropic effects than those in genes acting downstream. Studies have also demonstrated that immune system genes tend to exhibit higher rates of adaptive evolution, which have been attributed to their coevolution with pathogens $[37,39,50]$. Indeed, in our analysis, we detected a robust negative correlation between the rate of protein evolution and a gene's position in the Drosophila Toll and Imd signaling pathways, indicating that upstream genes experienced more relaxed selective constraints. A similar distribution of purifying selection has also been observed along the insulin/Tor pathways in vertebrates and Drosophila [51,52], the animal TLR signaling pathway [5], the $\mathrm{N}$-glycosylation metabolic pathway in primates [2], and the HOG signal transduction pathway in yeast [13].

Throughout our multivariate analysis, the correlation between gene position and $d N$ was significant, and given the results of path analysis, we see that pathway position can influence $\omega$ values through an effect on $d N$. Because $d N / d S$ is the measure of selection/constraint, $d N$ is actually the metric of selective pressure; these results overall demonstrated that gene position within the network was an important factor driving the polarity of selective constraints along Toll and Imd pathways. Although in the Caenorhabditis insulin/TOR signaling transduction pathway, the pattern of selective constraints was driven by expression level [1], and in the $\mathrm{N}$-glycosylation metabolic pathway in primates, connectivity was the main contributor [2] a study of animal TLR signaling pathway [5] agrees with our results in finding a negative relationship between evolutionary constraint and gene position. Because the Toll and Imd signaling pathways are homologous to the mammalian TLR 
Table 1 Spearman's rank correlation coefficient $(\rho)$ of the number of regulatory miRNA and the length of 3 '-UTR with $d N, d S$ and $\omega$

\begin{tabular}{ccccc}
\hline & & $\boldsymbol{d N}$ & $\boldsymbol{d S}$ & $\boldsymbol{\omega}$ \\
\hline $\mathrm{N}_{\text {miR }}$ & $\rho$ & $-0.563^{*}$ & $-0.360^{*}$ & $-0.507^{*}$ \\
& $\mathrm{P}$ & $<0.001$ & 0.023 & $<0.001$ \\
& lower & -0.702 & -0.593 & -0.660 \\
& upper & -0.357 & -0.111 & -0.283 \\
L $^{\prime}$ UTR & $\rho$ & $-0.502^{*}$ & $-0.377^{*}$ & $-0.503^{*}$ \\
& $P$ & $<0.001$ & 0.021 & $<0.001$ \\
& lower & -0.688 & -0.599 & -0.661 \\
& upper & -0.253 & -0.107 & -0.259 \\
\hline
\end{tabular}

This table is calculated based on all the genes involved in Toll and Imd pathways;

Lower and upper indicates the confidence intervals of the correlation;

${ }^{*}, P<0.05$ after the FDR correction at $\mathrm{q}=0.05$.

signaling pathway, which plays a vital role in animal innate immunity [32], the shared evolutionary pattern provides strong supporting evidence for our observations.

The negative correlation between the number of regulatory miRNAs and protein sequence evolution

In addition to the negative correlation between gene evolutionary rates and pathway position, we observed significant correlations between $N_{m i R}$ and the $d S, d N$, and $\omega$ values, and between $L_{3}{ }^{\prime} U T R$ and the $d S, d N$, and $\omega$ values. Given the significant correlation between $N_{m i R}$ and $L_{3^{\prime} U T R}$, to determine which was the main factor influencing evolutionary rates (or whether they both had an influence), partial analysis was performed. When controlling for $L_{3}{ }^{\prime} U T R$, the correlation between $N_{m i R}$ and $d N$ and between $N_{m i R}$ and $d S$ remained significant. However, when controlling for $N_{m i R}$, the significant correlation between $L_{3}^{\prime}$ UTR and $d N, d S$ and $\omega$ all disappeared, indicating that the correlation between gene evolutionary rates and $L_{3}$ 'UTR was mediated by the number of regulatory miRNAs. One possible explanation is that genes regulated by more miRNAs tend to have more molecular functions in different biological processes [53]. Consequently, these pleiotropic genes require more complex and precise regulation by miRNA [45].

In this study, we estimated different topological parameters that may influence the evolution of immunerelated gene within a network framework. Because $d N$, $d S$, and $\omega$ are subjected to many evolutionary pressures, there might be other topological factors that we did not consider in this study. Notably, to estimate the evolutionary pattern of genes in the Drosophila immune pathways Toll and IMD, the expression data we analyzed were gene expression levels after infection rather than constitutive expression. Compared with previous studies, we added two more topological parameters to our analysis (the number of regulatory miRNAs and the length of the 3 '-UTR) to improve our understanding of the impact of miRNAs on protein sequence evolution.

\section{Conclusion}

We found a polarity in the strength of purifying selection along the Drosophila Toll and Imd pathways, with the downstream genes being more conserved. Of all the immune genes investigated, two (Sphinx1 and Dnr1) exhibited signals of positive selection. Notably, we provided strong evidence to show that gene position within the pathway was an important parameter influencing protein sequence evolution within the Drosophila Toll and Imd innate immune response systems. Moreover, the negative correlation between protein sequence

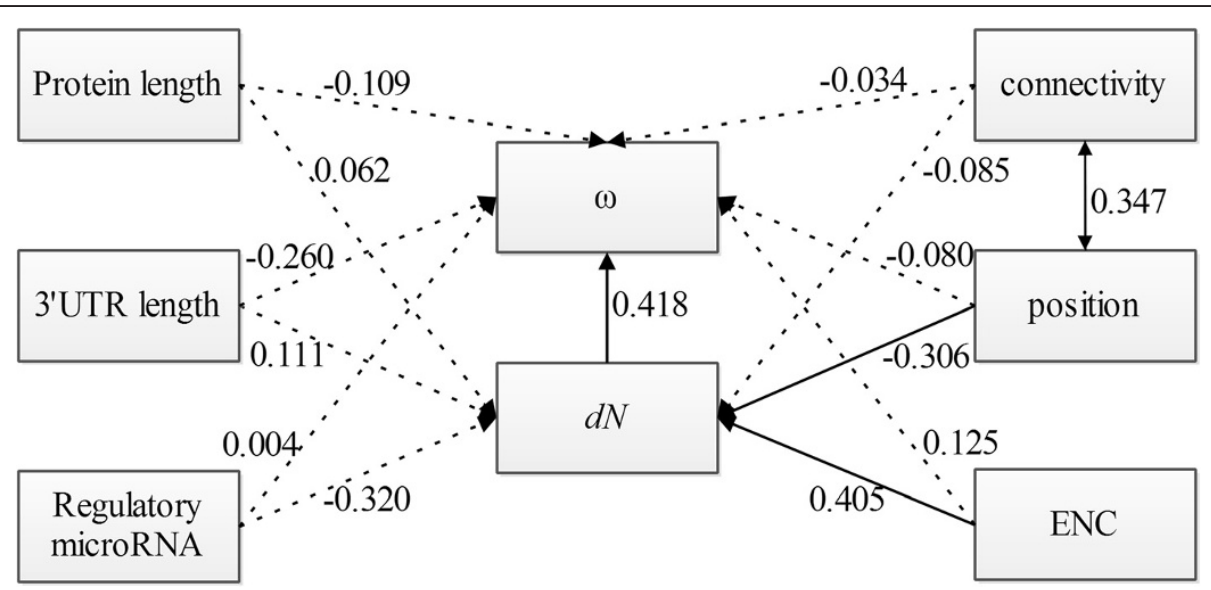

Figure 4 Path analysis of the relationships among codon bias (ENC), gene position, nonsynonymous substitution rate $(d N)$, $d N / d S$ ratio $(\omega)$, protein length, PPI, the number of regulatory miRNAs, and the length of the 3 '-UTR. Continuous and broken lines represent significant and nonsignificant relationships, respectively. Double-headed and single-headed arrows indicate the correlations and causal models assumed in the path analysis, respectively. Numbers on the arrows represent the standardized path coefficients ( $\beta)$. $d N$ and $\omega$ were considered endogenous variables, while the other variables were considered exogenous. 
evolution and the number of regulatory miRNAs confirmed that genes regulated by more miRNAs are likely to undergo stronger functional constraints, and therefore exhibit slower gene evolutionary rates. Further studies investigating the patterns of molecular evolution within different pathways will undoubtedly improve our understanding of natural selection in pathways and networks.

\section{Methods}

\section{Data collection}

By searching research articles and recent reviews [26,32,34-36,54-57], we compiled a list of immunerelated genes involved in the Toll and Imd pathways and depicted their relationships (Figure 1). We first downloaded the protein-coding DNA sequences (CDS) of all immune genes (Additional file 1: Table S6) in the D. melanogaster genome from FlyBase [58] and then identified orthologs of these genes in other Drosophila species (D. simulans, D. sechellia, D. yakuba, D. erecta, and $D$. ananassae) using the Database of Orthologous Groups (OrthoDB, http://cegg.unige.ch/orthodb6) [59]. If one gene had several transcripts, the longest was chosen as the ortholog for later analysis.

We recovered genes that were not annotated in OrthoDB through two BLAST steps. We first used protein sequences from $D$. melanogaster as queries and conducted BlastP searches against the whole genome of interest at NCBI, with a BLAST score $>150$ and length $>50$. The resulting sequence was then BLAST screened against the D. melanogaster genome. If the resulting protein sequence was the same as the original we obtained from FlyBase, we considered the resulting sequence an ortholog of the D. melanogaster one.

Some sequences appeared to have long deletions when aligned to orthologous genes; many such cases were artifacts rather than the true cases of deletion. Such sequences often contain stop codons when aligned, and consequently could not be analyzed in PAML. We recovered these incomplete sequences through several steps, as follows: we first conducted a Blat search on the UCSC genome browser [60], and the resulting sequence was used as a query to be predicted in the GeneWise tool with default parameters [61]. Predictions that did not contain frameshift mutations or internal stop codons were considered to be orthologs to D. melanogaster sequences. Alignments of these newly-annotated gene sequences are provided in Additional file 2.

\section{Multiple sequence alignment and phylogenetic analysis}

Multiple sequence alignment of the orthologous CDSs was conducted using MUSCLE [41] with default options. Using the resulting alignments, we reconstructed a Drosophila phylogeny with MrBayes [62], applying a mixed amino acid substitution model. Four chains with independent runs of
$10,000,000$ generations were performed to examine the parameter space. We sampled the trees every 1000 generations. The first 2,500 trees were discarded as burn-in. The analyses gave a well-supported topology that corresponded to the known relationships among Drosophila species [63]. This topology was used in subsequent codon-based tests of selection.

\section{Protein sequence evolution analysis}

The strength of selective pressures was examined by calculating $d S, d N$, and $\omega$ using the CODEML program in the PAML4.4 package [40]. Values of $\omega<1,=1$ and $>1$ indicate purifying selection, neutral evolution, and positive selection of the target gene, respectively. To avoid synonymous site saturation, which would prevent us from analyzing the more divergent CDS alignments [63], we limited this analysis to the six species in the melanogaster group (D. melanogaster, D. sechellia, D. simulans, $D$. ananassae, D. erecta, and D. yakuba). The F3 $\times 4$ codon frequency model [64] was applied throughout our codon-based analyses. We first conducted analyses using different models to evaluate changes in selective pressure: M0 (one ratio), M1a (nearly neutral), M2a (positive selection), M7 (beta), M8 (beta \& $\omega$ ), and M8a (beta \& $\left.\omega_{s}=1\right)$. We further tested whether some codon positions have undergone positive selection using a likelihood ratio test (LRT) [65] to compare models, specifically the M1a and M2a models, the M7 and M8 models, and the M8 and M8a models. To avoid false signals of positive selection, we also conducted a false discovery rate (FDR) test [66] controlling the $q$ value at 0.05 . We also performed our analyses using alignments obtained from PRANK $[42,43]$, which was reported to outperform other aligners in simulation [46], to explore discrepancies between our results and those of Sackton et al. [37].

\section{Network framework analysis}

Because the evolution of molecules within a network can be affected by multiple topological parameters, including gene position within the pathway, connectivity, protein length $\left(\mathrm{L}_{\mathrm{pro}}\right)$, codon bias (ENC), gene expression level (mRNA abundance), the number of regulatory miRNAs $\left(N_{m i R}\right)$ that target the gene, and the length of its 3'-UTR $\left(L_{3^{\prime} \text { 'UTR }}\right)$, we conducted a bivariate correlation analysis between these variables and the selective constraints parameters $(d S, d N$, and $\omega)$ applying Spearman's rank correlation coefficients $(\rho)$.

All the immune genes identified were directly involved in the Toll and Imd innate immune response signaling pathways, transducing signals from signal receptors (i.e., PGRPSA, PGRP-LC) to transcription factors (Dif, $d l, R e l$ ). We assigned a position to each gene according to its function within the pathway. Paralogous genes were assigned the same position. For instance, the nine Toll-related genes 
(Tl, 18w, Mstprox, Toll-4, Tehao, Toll-6, Toll-7, Tollo, Toll-9) were assigned the same position. Imd proteins accept signals from Gram-negative bacteria, whereas Toll receptors receive signals from Gram-positive bacteria and fungi. Both Toll and Imd receptors are located on cell membranes, so we assigned the Imd gene the same position as Toll. With signals from the activated Toll receptors, three molecules (MyD88, Tube, Pelle) are recruited to form a heterotrimeric complex, and they are closely connected with each other through two distinct death domains, thus their positions were considered the same as well. During microbe recognition, SPZ is cleaved by the activated enzyme SPE and then transduces signals to membrane surface receptors. Based on their close relationship, we attributed the same position to SPE and SPZ.

We also analyzed the Toll and Imd pathways separately; we observed similar results (Additional file 1: Table S7, Additional file 1: Table S8), which are not discussed further. The Toll and Imd pathways are both homologous to the mammalian TLR signaling pathway, which plays a vital role in animal innate immunity [32], genes in the Toll or Imd pathway are closely associated with each other (e.g., Traf6 in the Toll pathway regulates IкB kinase /NF-kB kinase, and Tak1 in the IMD pathway takes part in the formation of I $\kappa B$ kinase /NF- $\mathrm{kB}$ kinase). Additionally, expression profile analyses showed that both the Toll and IMD pathways can be up-regulated upon infection by the same pathogen $[67,68]$. Therefore, we preferred to analyze the two pathways as a unit.

\section{Multivariate analysis}

To better characterize the relationships among different topological parameters (gene position within the pathway, gene expression level, protein length, ENC, PPI, the number of regulatory miRNAs, the length of 3 '-UTR), we conducted partial analysis and path analysis. Through path analysis, the regression coefficients can be decomposed into direct and indirect correlations under a userdefined causal model. We therefore used path analysis to identify the main contributor constraining protein evolution within the network framework. For path analysis, $d N$ and $\omega$ were considered to be endogenous variables while the other variables were considered exogenous. To correct for the fact that the causal model in path analysis is user defined, the analysis was repeated with codon usage bias treated as an endogenous variable in addition to $d N$ and $\omega$.

All these analyses were conducted applying PASW statistical software. Codon usage bias values were calculated with DnaSP 5.10.01 [69]. Connectivity data (PPIs) of D. melanogaster were obtained from the BioGRID database [70]. Because the expression data of immune related genes upon infection was not available for all the
Drosophila species, we assumed that the relative expression levels of immune-related genes were conserved across species. Expression data of D. melanogaster after bacterial (Exp1) or fungal (Exp2) infection were obtained from De Gregorio et al. [67]. The number of regulatory miRNAs targeting the immune genes were predicted with Miranda 3.3 [44] using default parameters. To test the robustness of our predicted miRNA targets, we improved the Miranda score to 150.0, 160.0 and repeated the prediction. The resulting numbers of regulatory miRNAs were defined as $\mathrm{N}_{150}$ and $\mathrm{N}_{160}$, respectively.

\section{Additional files}

Additional file 1: Table S1. Summary statistics used in the multivariate analysis. Table S2. Phylogenetic analysis by maximum likelihood of the innate immune pathway genes (MUSCLE alignment). Table S3. Phylogenetic analysis by maximum likelihood of the innate immune pathway genes (PRANK alignment). Table S4. Bivariate correlations of different factors that may influence evolution of genes within a network (Toll and Imd pathways). Table S5. Bivariate correlations between connectivity and expression level of genes after infection. Table S6. Genes involved in the D. melanogaster innate immune pathway. Table S7. Bivariate correlations of different factors that may influence evolution of genes within a network (Toll pathway). Table S8. Bivariate correlations of different factors that may influence evolution of genes within a network (Imd pathway).

Additional file 2: Multiple sequence alignments of the Toll and IMD pathway orthologs across six Drosophila species. Alignments of the protein-coding sequences were performed using PRANK. Stop codons were removed from the ends of the alignments. Dots indicate the same nucleotide and "-"represents a missing nucleotide relative to sequences in the first row.

\section{Abbreviations}

MiRNA: microRNA; 3"-UTR: 3"-untranslated region; Dif: Dorsal-related immunity factor; FDR: False discovery rate; TLR: Toll-Like Receptor; PPI: Protein protein interaction; ENC: Effective number of codons; CDS: Protein coding DNA sequences.

\section{Competing interests}

The authors declare that they have no competing interests.

\section{Author's contributions}

FM and $\mathrm{MH}$ designed the study and drafted the manuscript together. $\mathrm{MH}$ SQ and XJS performed the research and analyzed the data. YFL, LMC and PJ analyzed the data. All authors have read and approved the final version of the manuscript.

\section{Acknowledgements}

This work was jointly supported by grants from the National Natural Science Foundation of China (No. 30970348), the Major Program of Natural Science Research of Jiangsu Higher Education Institutions (No. 12KJA180005), the Ph.D. Programs Foundation of Ministry of Education of China (No. 20113207110009) and the Priority Academic Program Development of Jiangsu Higher Education Institutions

\section{Author details}

'Laboratory for Comparative Genomics and Bioinformatics \& Jiangsu Key Laboratory for Biodiversity and Biotechnology, College of Life Science, Nanjing Normal University, Nanjing 210023, P. R China. ${ }^{2}$ The Key Laboratory of Developmental Genes and Human Disease, Ministry of Education, Institute of Life Science, Southeast University, Nanjing 210009, P. R China.

Received: 22 July 2013 Accepted: 6 November 2013

Published: 8 November 2013 


\section{References}

1. Jovelin R, Phillips PC: Expression level drives the pattern of selective constraints along the insulin/Tor signal transduction pathway in Caenorhabditis. Genome Biol Evol 2011, 3:715.

2. Montanucci L, Laayouni H, Dall'Olio GM, Bertranpetit J: Molecular evolution and network-level analysis of the $\mathrm{N}$-glycosylation metabolic pathway across primates. Mol Biol Evol 2011, 28(1):813-823.

3. Obbard DJ, Welch JJ, Kim K-W, Jiggins FM: Quantifying adaptive evolution in the Drosophila immune system. PLoS Genet 2009, 5(10):e1000698.

4. Ramsay H, Rieseberg LH, Ritland K: The correlation of evolutionary rate with pathway position in plant terpenoid biosynthesis. Mol Biol Evol 2009, 26(5):1045-1053

5. Song $X$, Jin $P$, Qin $S$, Chen $L, M a ~ F$ : The evolution and origin of animal toll-like receptor signaling pathway revealed by network-level molecular evolutionary analyses. PLoS One 2012, 7(12):e51657.

6. Stern DL, Orgogozo V: Is genetic evolution predictable? Science 2009, 323(5915):746-751

7. $Y-h$ Y, F-m Z, Ge S: Evolutionary rate patterns of the Gibberellin pathway genes. BMC Evol Biol 2009, 9(1):206

8. Yu H-S, Shen Y-H, Yuan G-X, Hu Y-G, Xu H-E, Xiang Z-H, Zhang Z: Evidence of selection at melanin synthesis pathway loci during silkworm domestication. Mol Biol Evol 2011, 28(6):1785-1799.

9. Lu Y, Rausher MD: Evolutionary rate variation in anthocyanin pathway genes. Mol Biol Evol 2003, 20(11):1844-1853.

10. Rausher MD, Lu Y, Meyer K: Variation in constraint versus positive selection as an explanation for evolutionary rate variation among anthocyanin genes. J Mol Evol 2008, 67(2):137-144.

11. Rausher MD, Miller RE, Tiffin P: Patterns of evolutionary rate variation among genes of the anthocyanin biosynthetic pathway. Mol Biol Evol 1999, 16(2):266-274.

12. Riley RM, Jin WI, Gibson G: Contrasting selection pressures on components of the Ras-mediated signal transduction pathway in Drosophila. Mol Ecol 2003, 12(5):1315-1323.

13. Wu X, Chi X, Wang $P$, Zheng D, Ding R, Li Y: The evolutionary rate variation among genes of HOG-signaling pathway in yeast genomes. Biol Direct 2010, 5(1):46.

14. Duret $L$, Mouchiroud D: Determinants of substitution rates in mammalian genes: expression pattern affects selection intensity but not mutation rate. Mol Biol Evol 2000, 17(1):68-070.

15. Pál C, Papp B, Hurst LD: Highly expressed genes in yeast evolve slowly. Genetics 2001, 158(2):927-931.

16. Drummond DA, Bloom JD, Adami C, Wilke CO, Arnold FH: Why highly expressed proteins evolve slowly. Proc Natl Acad Sci U S A 2005, 102 (40):14338-14343.

17. Lemos B, Bettencourt BR, Meiklejohn CD, Hartl DL: Evolution of proteins and gene expression levels are coupled in Drosophila and are independently associated with mRNA abundance, protein length, and number of protein-protein interactions. Mol Biol Evol 2005, 22(5):1345-1354.

18. Sharp PM: Determinants of DNA sequence divergence betweenEscherichia coli andSalmonella typhimurium: Codon usage, map position, and concerted evolution. J Mol Evol 1991, 33(1):23-33.

19. Fraser HB, Hirsh AE: Evolutionary rate depends on number of proteinprotein interactions independently of gene expression level. BMC Evol Biol 2004, 4(1):13

20. Fraser HB, Hirsh AE, Steinmetz LM, Scharfe C, Feldman MW: Evolutionary rate in the protein interaction network. Science 2002, 296(5568):750-752

21. Cheng C, Bhardwaj N, Gerstein M: The relationship between the evolution of microRNA targets and the length of their UTRs. BMC Genomics 2009, 10(1):431.

22. Sandberg R, Neilson JR, Sarma A, Sharp PA, Burge CB: Proliferating cells express mRNAs with shortened 3'untranslated regions and fewer microRNA target sites. Science 2008, 320(5883):1643-1647.

23. Stark A, Brennecke J, Bushati N, Russell RB, Cohen SM: Animal MicroRNAs confer robustness to gene expression and have a significant impact on 3'UTR evolution. Cell 2005, 123(6):1133-1146.

24. Taganov KD, Boldin MP, Baltimore D: MicroRNAs and immunity: tiny players in a big field. Immunity 2007, 26(2):133-137.

25. Sheedy F, O'Neill L: Adding fuel to fire: microRNAs as a new class of mediators of inflammation. Ann Rheum Dis 2008, 67(Suppl 3):iii50-iii55.

26. Tsakas $\mathrm{S}$, Marmaras V: Insect immunity and its signaling: an overview. ISJ 2010, 7:228-238.
27. Hetru C, Hoffmann JA: NF-KB in the immune response of Drosophila. Csh Perspect Biol 2009, 1(6)

28. Aggarwal K, Silverman NS: Positive and negative regulation of the Drosophila immune response. BMB Rep 2008, 41(4):267-277.

29. Qiu P, Pan PC, Govind S: A role for the Drosophila toll/cactus pathway in larval hematopoiesis. Development 1998, 125(10):1909-1920.

30. Halfon MS, Hashimoto C, Keshishian H: The Drosophila toll gene functions zygotically and is necessary for proper motoneuron and muscle development. BMC Dev Biol 1995, 169(1):151-167.

31. Belvin MP, Anderson KV: A conserved signaling pathway: the Drosophila toll-dorsal pathway. Annu Rev Cell Dev Biol 1996, 12(1):393-416.

32. Valanne $\mathrm{S}$, Wang JH, Rämet $\mathrm{M}$ : The Drosophila toll signaling pathway. J Immunol 2011, 186(2):649-656.

33. Lemaitre B, Nicolas E, Michaut L, Reichhart JM, Hoffmann JA: The dorsoventral regulatory gene cassette spätzle/toll/cactus controls the potent antifungal response in Drosophila adults. Cell 1996, 86(6):973-983.

34. Reumer A, Van Loy T, Schoofs L: The complexity of Drosophila innate immunity. Invertebr Surviv J 2010, 7(1):32-44

35. Kim T, Kim Y: Overview of innate immunity in Drosophila. J Biochem Mol Biol 2005, 38(2):121.

36. Wang L, Ligoxygakis P: Pathogen recognition and signalling in the Drosophila innate immune response. Immunobiology 2006, 211(4):251-261.

37. Sackton TB, Lazzaro BP, Schlenke TA, Evans JD, Hultmark D, Clark AG: Dynamic evolution of the innate immune system in Drosophila. Nat Genet 2007, 39(12):1461-1468

38. Schlenke TA, Begun DJ: Natural selection drives Drosophila immune system evolution. Genetics 2003, 164(4):1471-1480.

39. Obbard DJ, Jiggins FM, Halligan DL, Little TJ: Natural selection drives extremely rapid evolution in antiviral RNAi genes. Curr Biol 2006, 16(6):580-585.

40. Yang Z: PAML 4: phylogenetic analysis by maximum likelihood. Mol Biol Evol 2007, 24(8):1586-1591.

41. Edgar RC: MUSCLE: multiple sequence alignment with high accuracy and high throughput. Nucleic Acids Res 2004, 32(5):1792-1797.

42. Löytynoja A, Goldman N: An algorithm for progressive multiple alignment of sequences with insertions. Proc Natl Acad Sci U S A 2005, 102(30):10557-10562.

43. Löytynoja A, Goldman N: Phylogeny-aware gap placement prevents errors in sequence alignment and evolutionary analysis. Science 2008, 320(5883):1632-1635.

44. Betel D, Wilson M, Gabow A, Marks DS, Sander C: The microRNA. org resource: targets and expression. Nucleic Acids Res 2008, 36(suppl 1):D149-D153.

45. Chen SC-C, Chuang T-J, Li W-H: The relationships among microRNA regulation, intrinsically disordered regions, and other indicators of protein evolutionary rate. Mol Biol Evol 2011, 28(9):2513-2520.

46. Markova-Raina P, Petrov D: High sensitivity to aligner and high rate of false positives in the estimates of positive selection in the 12 Drosophila genomes. Genome Res 2011, 21(6):863-874.

47. Castoe TA, Jiang ZJ, Gu W, Wang ZO, Pollock DD: Adaptive evolution and functional redesign of core metabolic proteins in snakes. PLoS One 2008 3(5):e2201.

48. Flowers J, Sezgin E, Kumagai S, Duvernell D, Matzkin L, Schmidt P, Eanes W: Adaptive evolution of metabolic pathways in Drosophila. Mol Biol Evol 2007, 24(6):1347-1354

49. Jiggins FM, Kim K-W: Contrasting evolutionary patterns in Drosophila immune receptors. J Mol Evol 2006, 63(6):769-780.

50. Wang H-Y, Tang H, Shen C-KJ WC-I: Rapidly evolving genes in human. The glycophorins and their possible role in evading malaria parasites. Mol Biol Evol 2003, 20(11):1795-1804.

51. Alvarez-Ponce D, Aguadé M, Rozas J: Network-level molecular evolutionary analysis of the insulin/TOR signal transduction pathway across 12 Drosophila genomes. Genome Res 2009, 19(2):234-242.

52. Alvarez-Ponce D, Aguadé M, Rozas J: Comparative genomics of the vertebrate insulin/TOR signal transduction pathway: a network-level analysis of selective pressures. Genome Biol Evol 2011, 3:87.

53. He X, Zhang J: Toward a molecular understanding of pleiotropy. Genetics 2006, 173(4):1885-1891.

54. Silverman N, Paquette N, Aggarwal K: Specificity and signaling in the Drosophila immune response. Invert Surviv J 2009, 6(2):163.

55. Silverman N, Maniatis T: NF-KB signaling pathways in mammalian and insect innate immunity. Genes Dev 2001, 15(18):2321-2342. 
56. Marcu O, Lera MP, Sanchez ME, Levic E, Higgins LA, Shmygelska A, Fahlen TF, Nichol H, Bhattacharya S: Innate immune responses of Drosophila Melanogaster are altered by Spaceflight. PLoS One 2011, 6(1):e15361.

57. Huang HR, Chen ZJ, Kunes S, Chang GD, Maniatis T: Endocytic pathway is required for Drosophila Toll innate immune signaling. Proc Natl Acad Sci 2010, 107(18):8322-8327.

58. Crosby MA, Goodman JL, Strelets VB, Zhang P, Gelbart WM: FlyBase: genomes by the dozen. Nucleic Acids Res 2007, 35(suppl 1):D486-D491.

59. Kriventseva EV, Rahman N, Espinosa O, Zdobnov EM: OrthoDB: the hierarchical catalog of eukaryotic orthologs. Nucleic Acids Res 2008, 36(suppl 1):D271-D275.

60. Karolchik D, Baertsch R, Diekhans M, Furey TS, Hinrichs A, Lu Y, Roskin KM, Schwartz M, Sugnet CW, Thomas DJ: The UCSC genome browser database. Nucleic Acids Res 2003, 31(1):51-54.

61. Birney E, Clamp M, Durbin R: GeneWise and genomewise. Genome Res 2004, 14(5):988-995.

62. Ronquist F, Huelsenbeck JP: MrBayes 3: Bayesian phylogenetic inference under mixed models. Bioinformatics 2003, 19(12):1572-1574.

63. Clark AG, Eisen MB, Smith DR, Bergman CM, Oliver B, Markow TA, Kaufman TC, Kellis M, Gelbart W, lyer VN: Evolution of genes and genomes on the Drosophila phylogeny. Nature 2007, 450(7167):203-218.

64. Goldman N, Yang Z: A codon-based model of nucleotide substitution for protein-coding DNA sequences. Mol Biol Evol 1994, 11(5):725-736.

65. Whelan S, Goldman N: Distributions of statistics used for the comparison of models of sequence evolution in phylogenetics. Mol Biol Evol 1999, 16(9):1292.

66. Benjamini $Y$, Hochberg $Y$ : Controlling the false discovery rate: a practical and powerful approach to multiple testing. J R Stat Soc Ser B Methodol 1995, 57(1):289-300.

67. De Gregorio E, Spellman PT, Rubin GM, Lemaitre B: Genome-wide analysis of the Drosophila immune response by using oligonucleotide microarrays. Proc Natl Acad Sci 2001, 98(22):12590-12595.

68. Irving P, Troxler L, Heuer TS, Belvin M, Kopczynski C, Reichhart J-M, Hoffmann JA, Hetru C: A genome-wide analysis of immune responses in Drosophila. Proc Natl Acad Sci 2001, 98(26):15119-15124.

69. Librado P, Rozas J: DnaSP v5: a software for comprehensive analysis of DNA polymorphism data. Bioinformatics 2009, 25(11):1451-1452.

70. Stark C, Breitkreutz B-J, Reguly T, Boucher L, Breitkreutz A, Tyers M: BioGRID: a general repository for interaction datasets. Nucleic Acids Res 2006, 34(suppl 1):D535-D539.

doi:10.1186/1471-2148-13-245

Cite this article as: Han et al:: Evolutionary rate patterns of genes involved in the Drosophila Toll and Imd signaling pathway. BMC Evolutionary Biology 2013 13:245.

\section{Submit your next manuscript to BioMed Central and take full advantage of:}

- Convenient online submission

- Thorough peer review

- No space constraints or color figure charges

- Immediate publication on acceptance

- Inclusion in PubMed, CAS, Scopus and Google Scholar

- Research which is freely available for redistribution

Submit your manuscript at www.biomedcentral.com/submit
() Biomed Central 